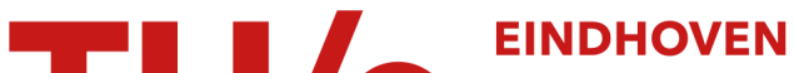 \\ UNIVERSITY OF \\ TECHNOLOGY
}

\section{Hard real-time reliable multicast in the DEDOS system}

Citation for published version (APA):

Alstein, D., \& Stok, van der, P. D. V. (1993). Hard real-time reliable multicast in the DEDOS system. (Computing science notes; Vol. 9317). Technische Universiteit Eindhoven.

Document status and date:

Published: 01/01/1993

\section{Document Version:}

Publisher's PDF, also known as Version of Record (includes final page, issue and volume numbers)

\section{Please check the document version of this publication:}

- A submitted manuscript is the version of the article upon submission and before peer-review. There can be important differences between the submitted version and the official published version of record. People interested in the research are advised to contact the author for the final version of the publication, or visit the $\mathrm{DOI}$ to the publisher's website.

- The final author version and the galley proof are versions of the publication after peer review.

- The final published version features the final layout of the paper including the volume, issue and page numbers.

Link to publication

\section{General rights}

Copyright and moral rights for the publications made accessible in the public portal are retained by the authors and/or other copyright owners and it is a condition of accessing publications that users recognise and abide by the legal requirements associated with these rights.

- Users may download and print one copy of any publication from the public portal for the purpose of private study or research.

- You may not further distribute the material or use it for any profit-making activity or commercial gain

- You may freely distribute the URL identifying the publication in the public portal.

If the publication is distributed under the terms of Article 25fa of the Dutch Copyright Act, indicated by the "Taverne" license above, please follow below link for the End User Agreement:

www.tue.nl/taverne

Take down policy

If you believe that this document breaches copyright please contact us at:

openaccess@tue.nl

providing details and we will investigate your claim. 
Eindhoven University of Technology

Department of Mathematics and Computing Science

Hard Real-Time Reliable Multicast in the DEDOS system

by

D. Alstein and P. van der Stok

$93 / 17$

Computing Science Note 93/17

Eindhoven, June 1993 


\section{COMPUTING SCIENCE NOTES}

This is a series of notes of the Computing Science Section of the Department of Mathematics and Computing Science Eindhoven University of Technology. Since many of these notes are preliminary versions or may be published elsewhere, they have a limited distribution only and are not for review.

Copies of these notes are available from the author.

Copies can be ordered from:

Mrs. M. Philips

Eindhoven University of Technology

Department of Mathematics and Computing Science

P.O. Box 513

$5600 \mathrm{MB}$ EINDHOVEN

The Netherlands

ISSN 0926-4515

All rights reserved

editors: prof.dr.M.Rem

prof.dr.K.M.van Hee. 


\section{Hard Real-Time Reliable Multicast in the DEDOS system}

\section{Dick Alstein}

Eindhoven University of Technology

Department of Mathematics and Computer Science

Address: P.O.Box 513, 5600MB Eindhoven, The Netherlands

E-mail: wsinda@win.tue.nl, wsstok@win.tue.nl

May 14, 1993 


\begin{abstract}
Two protocols for reliable multicast in a multiprocessor system are presented. The protocols use mailboxes located in common memory to exchange messages, instead of relying on communication channels. The mailboxes are replicated for reliability.

The protocols have bounded completion time, making them suitable for use in hard realtime systems. Multiple source ordering is imposed on the messages that pass through a mailbox. The protocols are shown to be tolerant of memory crashes, and of process or processor failures. The basic protocol tolerates only crash failures of processes, while the second protocol can also handle timing failures.

The protocols can be extended so that multicast group membership is dynamic.
\end{abstract}

Keywords: real-time, fault tolerance, multiprocessors, shared memory, group communication, crash and timing failures. 


\section{Introduction}

Multiprocessor systems often offer broadcast and multicast services. Broadcast enables a processor to send a message to every other processor in the system. Multicast is similar, but limits the distribution to a specified subset of processors, a multicast group. When multiprocessor systems need fault tolerance in order to work reliably, these services are also required to be reliable, i.e. they should guarantee delivery despite failures such as message loss and processor crashes. As it is virtually impossible to ensure receipt of a message by a failed destination, a broad- or multicast is called reliable if it delivers a message either at every intact (i.e. non-failed) destination, or at none a.t all. In order to exclude the trivial solution of refusing to receive any message, it is also required that if the sender is intact, its messages will be received by every intact destination.

In addition to reliability, broadcast and multicast services usually provide some form of message ordering. Garcia-Molina and Spauster [Garc89] distinguish three types of ordering. In order of increasing strength these are single source ordering, multiple source ordering and total ordering. Single source ordering can be established easily by numbering messages at the sender. However, many applications demand stronger ordering. Multiple source ordering and total ordering are harder to achieve, and at higher overhead cost.

In this paper, two protocols for reliable multicast are presented. Not only are the protocols required to be reliable, they must also impose multiple source ordering on the messages. This implies that all members of a given multicast group receive incoming messages in the same order, even when these have originated from several senders. Total ordering is considered too strong for general use, since it requires all ordering of messages between different (possibly unrelated) groups.

In addition, the protocols are intended to work in a harl real-time system. In such a system, the correctness of a computation depends not only on its logical result, but also on the time at which it is delivered. Each computation is accompanied by a deadline, which must be met at all cost, because violation of a deadline has catastrophic consequences. As a result of these deadlines, services in a hard real-time system are required to have a bounded completion time: there must be a known maximum delay between the invocation and the completion of a service.

Thus, our protocols for reliable multicast must satisfy the following protocol requirements:

Requirement 1 Consensus: A message is delivered either at every intact destination, or at none at all.

Requirement 2 Validity: A message from an intact sender is delivered at every intact destination.

Requirement 3 Ordering: There is multiple source ordering on the messages.

Requirement 4 Bounded time: There are known maximum times for the completion of send and receive operations.

Several algorithms for reliable broad- and multicast are known from the literature. ${ }^{1}$ Chang and Maxemchuk [Chan84] used a. central processor called the token site, which gathers all

\footnotetext{
${ }^{1}$ In some publications, the term atomic is used instead of reliable.
} 
messages, imposes a total ordering, and redistributes them. In case the central site fails, a token-passing protocol is used to elect a new central site. Birman and Joseph [Birm87] devised a fully decentralized protocol, working in two phases. In the first phase a message is disseminated to all processors and acknowledged, in the second phase a total order is established, after which the messages can be delivered to their destinations. Cristian et al. [Cris89] developed a broadcast protocol for systems with bounded communication delays. Their protocol is based on message diffusion, which poses no demands on network topology. Timestamps on the messages, read from synchronized clocks, supply a total ordering.

These algorithms were all designed for distributed systems, where the only way to exchange information between processors is by sending messages along communication channels. By contrast, the protocols presented here address the problem for multiprocessor systems with common memory, as is the case for the Dependable Distributed Operating System (DEDOS, [Stok92]). Inside the multiprocessor a block of memory is allocated for communication purposes; senders can access it to deposit their messages, which receivers can then read. This concept is known as a mailbox. The most important advantage of using mailboxes over message-passing communication is location independence: senders only need to know the location of the mailbox, in order to reach the receivers. This facilitates the implementation of process migration and dynamic group membership.

Unfortunately, using mailboxes also has an important disadvantage: it introduces reliability problems, because a mailbox forms a single point of failure. If the part of memory that the mailbox is located in fails, the messages stored in that part of memory are lost. It is likely that these messages have already been read by some destinations, but not by others. Therefore, requirement 1 is not met.

The protocols presented here solve these reliability problems by replicating the mailbox. A mailbox consists of a number of replicates, called copies. Senders store their message in each copy. It is assumed that it is possible to locate the copies in separate parts of common memory, so that their failure probabilitics are independent. Thus, the probability that a message is irretrievably lost can be reduced to a negligible value by increasing the number of copies.

The protocols differ in the classes of failure that they can tolerate. The first protocol assumes that only crash failures occur. The second protocol is an extension of the first; it can also handle timing failures.

The remainder of this paper is structured as follows. Section 2 lists the assumptions about the distributed system for which the protocols are intended. In Section 3, the protocols are described. In Section 4, the performance of the protocols is discussed, and a comparison with existing protocols is made. Possible extensions of the protocols are discussed in an appendix.

\section{System architecture and failure assumptions}

In this section, the system requirements are listed. They describe the hardware, operating system services, and failure assumptions that are needed for a correct functioning of the protocols.

\subsection{Hardware}

The system hardware consists of a number of processing elements called processors. Each processor contains one CPU, private memory to store local data, a real-time clock, and some 
interface hardware. The system contains common memory, with every memory cell being accessible from each processor. Access to common memory is governed by an arbitration mechanism, ensuring fairness and avoiding collisions. Typically, basic operations on memory, such as reading/writing one cell are executed in a serial order, so that memory access of one processor does not interfere with others.

\subsection{Operating system services}

On each processor a number of application processes are located. The processes run concurrently, and are identified by a system-wide unique process identifier $(P I D)$. Each processor also runs an operating system kernel. The kernels on all processors cooperate to provide system services. An application process issues calls to the local kernel to ask for system services. Our protocols assume the presence of three services: clock synchronization, mailbox server, and process membership.

The clock synchronization service provides closely synchronized clocks. The clocks on any pair of intact processors in the system differ by less than a fixed maximum $\epsilon$. Clock values are monotonic and increasing, i.e. subsequent readings of a clock yield different, higher values.

The mailbox service controls the creation and removal of majlboxes, and the access of processes to these mailboxes. Every mailbox has a system-wide unique name. A process that wants access to a mailbox, performs a call to the mailbox server, which returns a reference to the mailbox. When communication is no longer nceded, the process disconnects from the mailbox. Although this service is closely related to the multicast protocols, the algorithms underlying its functioning are beyond the scope of this paper (cf. [Dijk92]).

The process membership service is respousible for gathering and redistributing information on start, termination and failure of processes. The multicast protocols, rather than diagnosing and treating failures themselves, transfer information on detccted failures to the membership service [Clae92].

\subsection{Failure hypothesis}

Failure classification Any algoritlm that claims to be functioning in the presence of failures will have to specify the type and number of failures it can tolerate. For this purpose, we adopt the failure classification given in [Cris91], which is summarized below.

Each system component is required to respond to inputs in a way that is consistent with its specification. This specification prescribes not only the output that should be given, but also the time interval in which the output should be delivered. If the behavior of a component is according to its specification, it is called intret. Otherwise, a failure has occurred; the component is said to have failed.

An omission failure occurs when a component does not respond to an input. If, after a first omission failure, it also does not respond to subsequent inputs until it is restarted, it is said to suffer a crash failure. A timing failure occurs when the response is functionally correct, but given outside the time interval specified. If the response is given too early, we speak of an early timing failure, if it is too late we have a performance failure. A response failure occurs when the response is incorrect, either in the output value, or in the state transition.

When a component exhibits only crash fajlures, it is called fail-silent: either its output is correct, or it does not give any output at all, and remains silent until restart. 
The classes of crash, omission, timing and response failures are, in this order, of increasing generality. Algorithms tolerant of such failures will have increasing cost and complexity in the same order.

Failure hypothesis We can now state our assumptions about the failures that the protocols can tolerate.

- Processors in the system may suffer crash or timing failures.

- Application processes and the operating system kernel on a processor may also fail. A processor or kernel failure is taken to be equivalent to the failure of all processes located on that processor. A process failure does not affect other processes.

- Common memory is assumed to be fail-silent. The memory is assumed to be subdivided into modules, in such a way that the modules have independent failure probabilities. Memory failures must be detectable by processes reading it: if a read operation on common memory by a process is unsuccessful, the process is notified of a crash (e.g. by means of an exception mechanism). Subsequent reads from any processor to that module yield the same result.

It is assumed that there is an upper bound to the time that is needed for a read or write in common memory, regardless of whether the memory is intact or not.

- It is assumed that at most $f$ copies of a mailbox fail. Thus, if more than $f$ copies are created, it is certain that at least one of them remains intact.

\section{Protocol description}

In this section, the multicast protocols are described. The first protocol is capable of handling processor crash failures, but is not tolerant of processor timing failures. The second protocol also tolerates timing failures. But to begin, we introduce some notations and definitions.

A multicast group is a subset of the set of processes in the system. A message sent to a multicast group must be received by each intact process that is a member of that group. The exchange of messages is done through a mailbox $M$. The mailbox is replicated; it consists of $n$ copies, with $n>f$ so that at least one copy will remain intact (since by assumption at most $f$ copies fail). The $n$ copies of $M$ are denoted by $M_{1}, M_{2}, \ldots, M_{n}$. An arbitrary copy is indicated by $M_{i} . M_{i}$ can be either intact or failed. Since every multicast group has exactly one mailbox, we may alternately speak of "mailbox" and "multicast group" to indicate the same object. In the remainder of this paper, a mailbox will denote a set of copies.

A process that is a member of the multicast group is called a receiver. The number of receivers (the size of the multicast group) is denoted by $N$. It is assumed that at most $t$ of these receivers have experienced a failure.

A sender is a process that is able to send messages to the multicast group. A sender is not necessarily also a receiver: any process can become a. sender, by asking the mailbox server for a reference to the mailbox.

A send operation consists of writing a message into the mailbox by a sender. Likewise, a receive is done by a receiver reading the next message from the mailbox. After reading it, the receiver decides on the message: it may either accept or reject it. Rejection occurs. when the receiver regards the message as invalid, otherwise the message is accepted. 


\subsection{General characteristics}

Availability-flag Senders write their messages into each copy, so that a message remains present in the mailbox despite the crash of all but one of the copies. However, it is also necessary that receivers do not accept a message that is present in a copy unless it is certain that the message has been written in all copies. To signal this, each message is tagged with an availability flag (a-flag). The sender first writes the message in every copy, and then sets all a-flags. If a receiver finds an a-flag set, the message is guaranteed to remain available to later receivers, so that it can safely be accepted.

Time bounds There are upper bounds on execution times for operations on one copy. We define the following constants:

$\delta_{s} \quad$ maximum time needed to write a message

$\delta_{f} \quad$ maximum time needed to set the a-flag

$\delta_{d} \quad$ maximum time needed to decide upon a message

$\delta_{r} \quad$ maximum time needed to read a message

$\delta_{f d}$ maximum time needed to set the d-flag and delete a message

These are the times needed for completing an operation on a single copy. Based on these constants, it is possible to derive upper bounds for the execution time of operations on the whole mailbox. These constants are $\Delta_{s}$ and $\Delta_{r}$ for a send and receive operation, respectively.

Two more requirements must be met for the existence of these upper bounds. First, processes must not be preempted during the execution of an operation. Second, there must be a maximum message size, as sending and receiving involves copying of the message contents to and from common memory.

Ordered copies Basically, the copies of the mailbox are organized as a chain. Any operation on the mailbox is first executed on $M_{1}$, then on to $M_{2}$, and so on.

The number of copies is constant; the copies are created and allocated at mailbox creation time, and no new copies are added thereafter. Copies may crash, but are not replaced.

Timestamps Each message in the queue is accompanied by a timestamp. The timestamp indicates the time at which the Send operation started. The sender reads it from its local clock. Messages are placed in the queue in the order of their timestamp. The sender PID is added as a suffix, to break ties in case two timestamps are equal.

Receivers use the timestamp to determine whether a certain message can already be read: they are only allowed to read it if the timestamp is "old enough", i.c. if the sending should have been completed. This can be secn by the timestamp: a message with timestamp $\tau$ can be read if the local clock on the receiver is past time $\tau+\Delta_{s}+\epsilon$.

Decision field and decision flags To ensure unanimity of decisions, each message in the quene has a decision field. Once a receiver has taken a final decision, this decision is written into the decision field. The atomicity of writing this field (in a single copy) guarantees a consistent decision by all intact receivers.

Additionally, every message is accompanied by a list of decision flags ( $d$-flags). There is one d-flag for every receiver in the multicast group. The flag signals that the corresponding receiver has read the message and decided upon it. The d-flags serve two purposes. First, to 
signal that a message has been read by all receivers, so that it can be safely removed. Second, to enable recovery actions in case of a receiver crash (see Section 3.3.2).

Separate reading and deciding Taking a decision about a message is decoupled from reading the message data. Receivers first decide upon the message, and write this decision into the appropriate data structures in every copy. After that, they can start reading the message data itself.

This has the advantage that the latter task, which may involve copying large amounts of data to private memory, is only executed on one copy. This may be postponed until a convenient time. It is guaranteed that the message will remain available in the mailbox, because receivers set the d-flag only after they have read the message.

Buffer size At present, the size of the mailbox buffers (i.e the amount of memory available for all data) is assumed to be sufficient, so that no buffer overflow will occur. For a further discussion, see Section 3.3.2.

\subsection{Data structures}

The following data structures are used by both protocols.

- Each copy of the mailbox contains a receiver list, containing the PIDs of the receivers that form the multicast group, and a message queue, contajning the messages in transmission.

- An entry in the message queue consists of:

- The sender PID.

- The timestamp.

- The decision field $\mathrm{D}$.

- A list of d-flags, one for each receiver in the multicast group, indicating that the receiver has decided upon the message.

- The a-flag, indicating whether the sending of the message has been completed.

- The message data.

The decision field and the flags contain values from the set $\{\perp, 0,1\}$, where $\perp$ means undefined. For D and the d-flags, a value of 0 means that the message has been rejected, and when the flag is 1 the message was accepted. An a-flag is 1 if the sender has completed the writing, and 0 if the sender has started but not (yet) finished. An a-flag is never set to $\perp$.

- Each connected process stores the locations of all copies of the mailbox. The copies are ordered, and this ordering is the same in every sender or receiver. Additionally, each receiver stores the timestamp of the most recent message it has read, to decide which message to read next. 


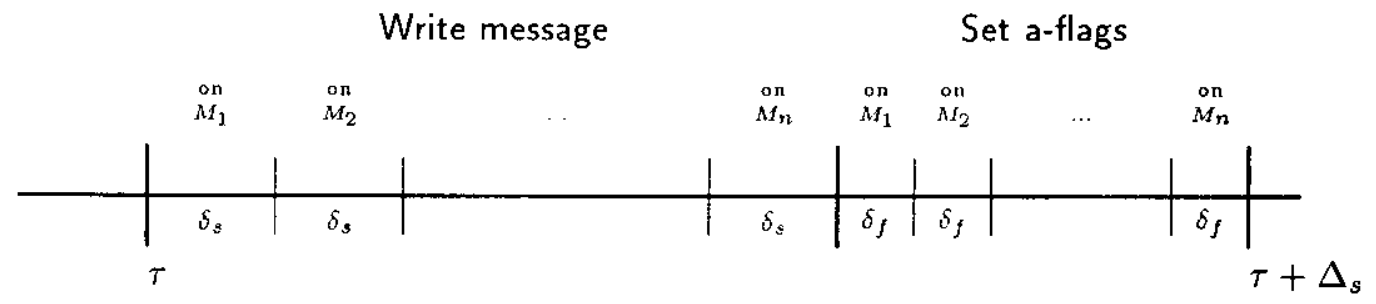

Figure 1: Timing diagram of a typical send operation with timestamp $\tau$.

\subsection{First protocol}

\subsubsection{Protocol description}

Send procedure Scnding a message consists of two phases. First, the process determines the timestamp, and inserts the message into the queue of each copy. D is initialized to 1 , and a d-flag is created for every receiver in the receiver list. The message is at first marked as not available (i.e. the a-flag is cleared). Second, when every mailbox copy has been written, the sender sets the a-flag in each copy.

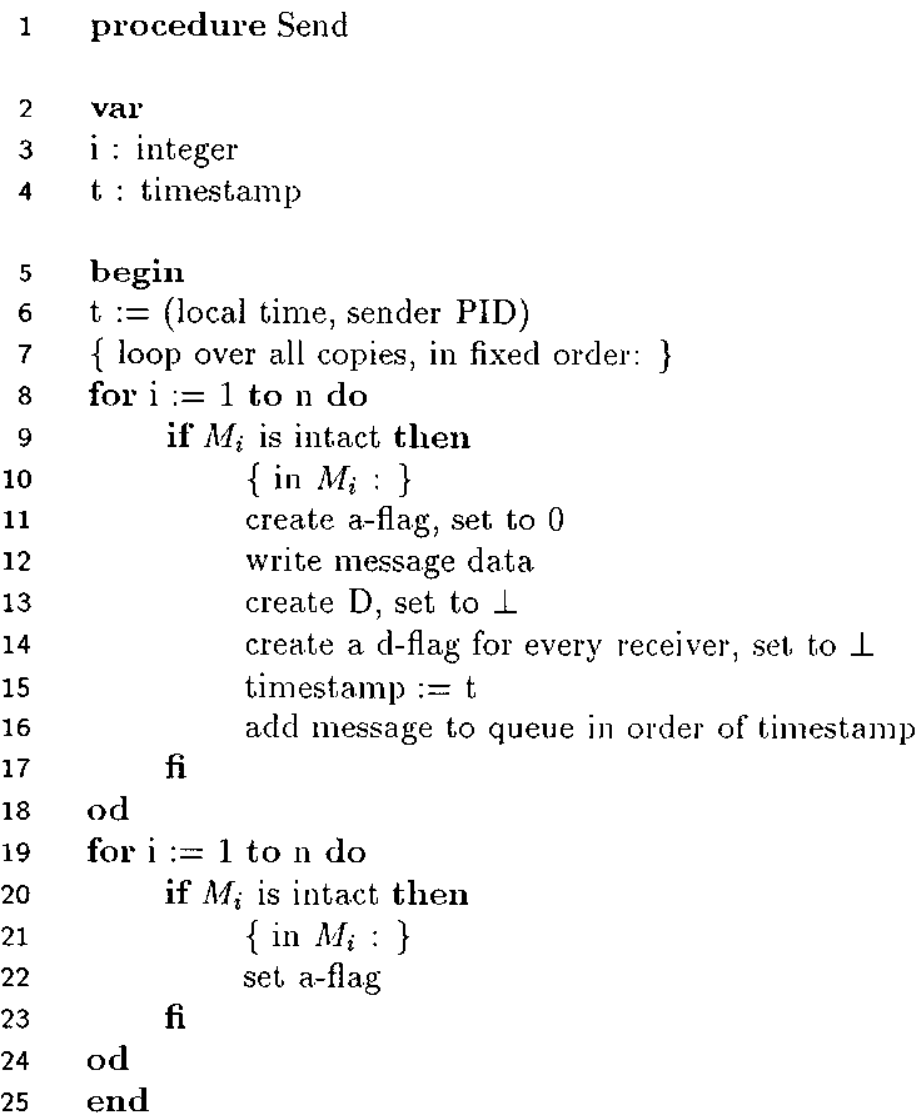




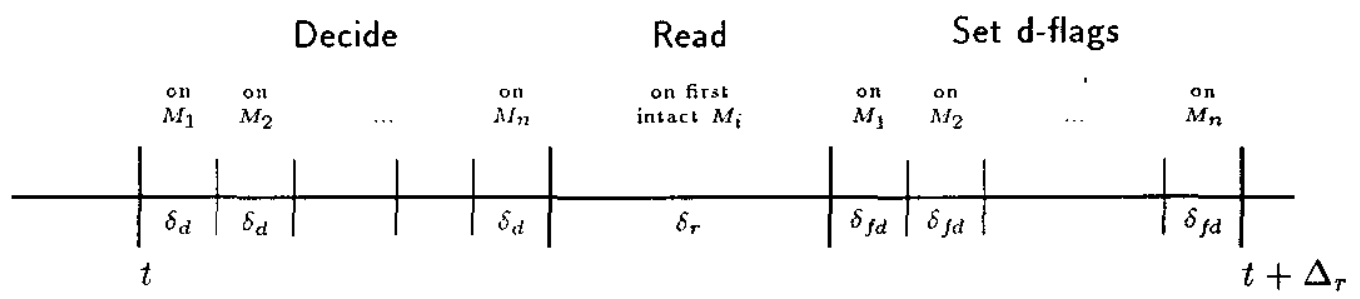

Figure 2: Timing diagram of a typical receive operation starting at time $t$.

Receive procedure When a receiver is ready to receive the next message, it checks the message queue in the first intact copy. If there are no new messages, it waits for a certain period $\delta$, then checks again.

When it has found an unread message, the receiver has to decide upon the message. First, it checks if the decision field has already been set by another receiver. If this is the case, that decision is followed. Otherwise, the decision is based upon the state of the a-flag. If the a-flag is set, the sender has completed writing the message in all copies, and the message is accepted. If the a-flag is cleared, the sender must have crashed before it could finish writing, and the message is rejected.

The receiver stores the decision in a local variable (firstD), and writes it in the decision field in all subsequent intact copies.

If it has decided to accept, it may read the message, After that, the receiver sets the d-flag according to the decision, in every copy. If all other d-flags have also been set, all receivers have read the message, and so it is removed by the receiver.

1 procedure Receive

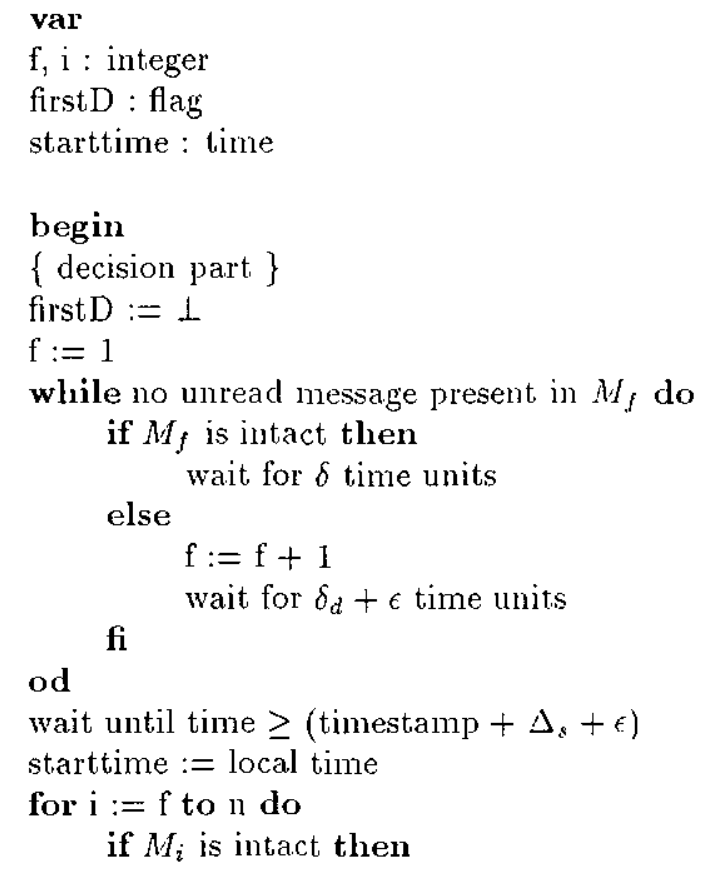




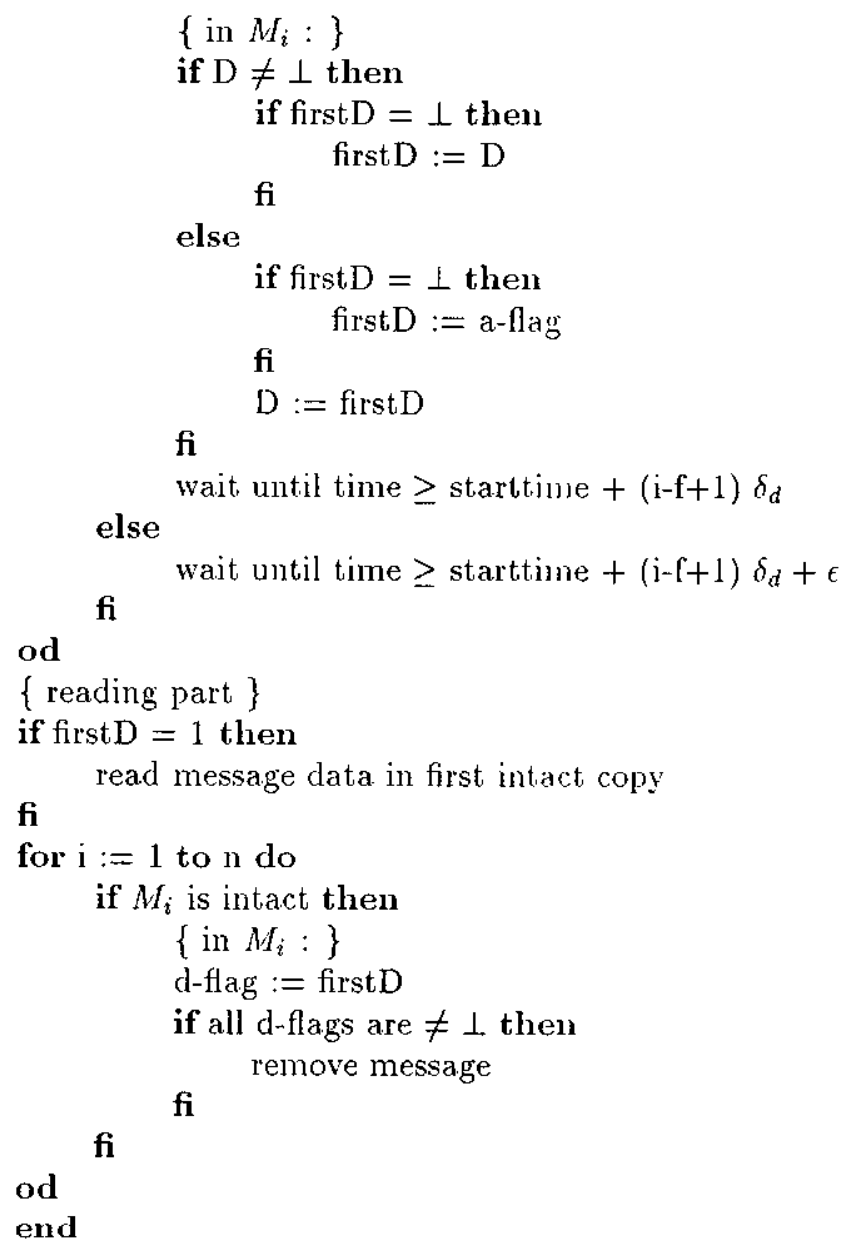

The Receive procedure has a timed execution: a fixed time interval of $\delta_{d}$ is reserved for each copy. After all operations on a copy have been done, the receiver waits until the end of the interval.

Notice that if a copy is found to have failed, an extra time delay of $\epsilon$ is introduced. This is done to avoid race conditions. Consider the following scenario: a sender crashes after setting the a-flag in $M_{1}$. The first receiver reads the message, and decides to accept it. Just after that decision, $M_{1}$ crashes. A second receiver notices the crash. If no delay were introduced, this second receiver might "overtake" the first one, and decide to reject the message (since the a-flag was not set in $M_{2}$ ).

If all $d$-flags are set, the receiver removes the message. The check and the subsequent remove action should be done atomically. Otherwise, if several receivers execute this at the same time, a message may be removed more than once, or not at all.

\subsubsection{Garbage collection}

So far, the treatment of failures has not been discussed. Althougl the occurrence of a process or memory crash does not directly endanger the consistency of a mailbox, it could result in buffer overflow, if no measures are taken. If a receiver crashes, not all d-flags will be set, so that messages could remain in the mailbox indefinitely. Moreover, the receiver is still in the 
receiver list, causing the same problem to occur for oncoming messages. As there is no timeout for receivers, the protocol can not detect a crash. It relies on other sources for reports of receiver crashes.

The task of garbage collection, i.e. the release of buffer space that is no longer necessary, is left to the mailbox server. If a receiver crash is reported, the mailbox server updates the receiver list, marking the receiver as crashed. Then it sets the d-flag in all messages in the queue that were not yet read by the receiver. ${ }^{2}$

The size of buffers in the mailbox needs to be adapted to the message load and the speed of crash detection in the system, so that the probability of buffer overflow is negligibly low.

\subsubsection{Correctness of the first protocol}

Theorem 1 The first protocol satisfies the four requirements of consensus, validity, ordering and bounded time.

\section{Proof:}

Consensus: Let $A$ and $B$ be receivers that have both completed the receiving of a message. Let $M_{x}$ and $M_{y}$ be the first intact copies that they found, i.e. in which their decision was made, and let $x \leq y$ without loss of generality. We distinguish six cases:

1. A and $B$ both base their decision upon the a-flag, and $x=y$.

When the recejvers start reading a message, the sender has either completed the sending, or crashed. Either way, the value of the a-flag does not change thereafter. Both receivers take the same decision.

2. $A$ and $B$ both base their decision upon the a-fang, and $x<y$.

This is an impossibility: $B$ found that $M_{x}$ had crashed, and thus must have accessed it after $A$ did (since $A$ found the copy intact). According to the algorithm, $B$ introduced an extra delay and therefore accessed $M_{y}$ after $\mathrm{A}$ (or another receiver) had written its decision in the decision field in $M_{y} . B$ must follow $A$ 's decision.

3. A bases its decision upon the a-flag, $B$ upon the decision field, and $x \leq y$. $B$ follows the decision thatt was written in the decision field by $A$ or by another receiver $C$, which took the same decision as $A$ (case 1 ).

4. A bases its decision upon the decision field, $B$ upon the a-flag, and $x<y$. By an argument analogous to case 2, this can be shown to be impossible.

5. $A$ and $B$ both base their decision upon the decision field, and $x=y$.

Once a value has been written into the decision field, it is not overwritten. Both receivers take the same decision.

6. $A$ and $B$ both base their decision upon the decision ficld, and $x<y$.

$B$ follows the decision that was written in the decision field by $A$ or by another receiver $C$, which took the same decision as $A$.

Validity: If the sender is intact, it will complete the Send operation within $\Delta_{s}$ time units. Therefore, all receivers start reading after the a-flag has been set; the message will be accepted.

\footnotetext{
${ }^{2}$ These actions are similar to the execution of a. Leave operation, as described in Appendix A.1.
} 
Ordering: Messages are stored in the queue, and read from it, in the order of their timestamps. Because of the receive delay of $\Delta_{s}+\epsilon$ after the timestamp, it is not possible that while one message is being read, another one with an earlier timestamp is inserted in the queue.

Bounded time: Neither senders, nor receivers are required to wait for other processes during an operation. Since single instructions have a bounded execution time, Send and Receive operations are also completed in bounded time.

\subsection{Second protocol}

\subsubsection{The first protocol can not tolerate timing failures}

Although the first protocol is tolerant of late timing failures, it does not tolerate early timing failures. A receiver with a fast clock may reject a message from an intact sender (since the a-flag is not yet set), and force all other receivers to reject the message as well. This violates the validity requirement (page 1 ).

In this way, a fast receiver can effectively block all communication in a multicast group. This is especially a problem when process replication is used as a means of increasing the reliability. Multicast communication would be ideally suited to distribute the input to the process among the replicas. However, if communication can be inhibited by a timing failure of a single replica, the replication would be useless.

The cause underlying this deficiency is that in the first protocol, a receiver is not required to read a message within a certain time interval after the sending. It may start receiving at any time. As a consequence, it is unable to detcrmine whether another receiver is too fast (and has failed), or is intact (but started receiving at an earlier time). In the latter case, the decisions should be the same, in the former, the decision of the first receiver can be ignored.

If the Receive procedure is to be modified to tolerate timing failures, the starting procedure should be coordinated. That is, intact reccivers must start receiving a message with timestamp $\tau$ before time $\tau+c$, for some constant $c$. One way to achieve this is to let receivers periodically poll the message queue for new messages. Another way is by using an off-line schedule, as found in the DEDOS system. Such a schedule prescribes the times at which operations must be started, so that it is known beforehand when a send operation should be completed. Our algorithm is valid for both approaches. In the description below, let $p$ be the "polling delay", i.e. the maximum time for a receiver to detect the presence of a message. If an off-line schedule is used, $p=0$.

Quorum The idea behind the second protocol is to use a quorum. Receivers take a tentative decision, based on the a-flag. They write this decision in common memory, and increase a counter. After a sufficient number of receivers has done so, the decision is final. Late receivers simply follow this decision.

The minimal size $\mu$ of a quorum for the second protocol is a majority of the receivers: $\mu=\left\lceil\frac{N+1}{2}\right\rceil$. The protocol is only correct if a quorum contains at least one intact receiver, i.e. $t<\mu$. 
Expiry time Destinations must postpone processing a message until it is certain that no other message with an earlier timestanp will be accepted. A problem with timing failures is that slow senders may keep adding messages with early timestamps to the queue. In the second protocol, this is countered by defining an expiry time $\lambda$. Before contributing to the decision, receivers check if their local time is past $\tau+\chi$. If not, they increase a counter. Receivers are only allowed to change the decision if a majority of the receivers has seen the message before the expiry time (i.e. the counter is $\geq \mu$ ). If the sender is too slow, most receivers will observe that their clock is past the expiry time. Thus, the decision will not be modified, and the message is rejected.

The value of $\chi$ should be such that, for a message from an intact sender, intact receivers wait long enough to establish a quorum: $\chi \geq \Delta_{s}+p+n \delta_{d}+2 \epsilon$.

Scanning the message queue The possibility of slow senders rajses another problem. Intact receivers that scan the queue for the "earliest" unread message may not come up with the same message. If they simply wait for a quorum to be formed, the system might even deadlock. Instead, receivers should keep scanning the message queue for newly added messages, while waiting until a delay time has passed.

\subsubsection{Protocol description}

The decision field is extended to a 4 -tuple (nrd, nxp, LD, D). The 4-tuple is initialized by the sender to $(0,0,0, \perp)$. The integer nrd is a counter for the number of receivers that have taken part in the decision. Likewise, nxp counts the receivers that saw the message before the expiry time. The flag LD represents the group decision that is being made locally, i.e. in this copy. It may be changed by a recciver as long as nrd is less than $\mu$. D is a flag that contains a final decision that has been made previously. If a receiver has witnessed a decision in a previous copy, it writes that decision into the $\mathrm{D}$ field. This decision overrides the value of LD: if $\mathrm{D} \neq \perp$, the decision in this copy is determined by $\mathrm{D}$, otherwise it is determined by LD. If $\mathrm{D}$ is still equal to $\perp$ when a quorum has been formed, the value of $\mathrm{LD}$ is copied into D.

The Send procedure is the same as in the first protocol. Only the Receive procedure is different.

The Receive procedure must ensure that it is impossible for a fast receiver to cause the rejection of a message from an intact sender. To achieve this, the private decisions of receivers are compared (the set of flag values is ordered, with $\perp<0<1$ ). A receiver is only allowed to write its decision into the LD field if it is larger than the current value of LD (i.e. "reject" may be overwritten with "accept").

1 procedure Receive

2 var

$3 \quad \mathrm{i}$ : integer

4 d, firstD : flag

5 begin

$6 \quad$ decision part

7 firstD $:=\perp$

8 wait until time $\geq$ (timestamp $+\Delta_{s}+\epsilon+p$ ) 


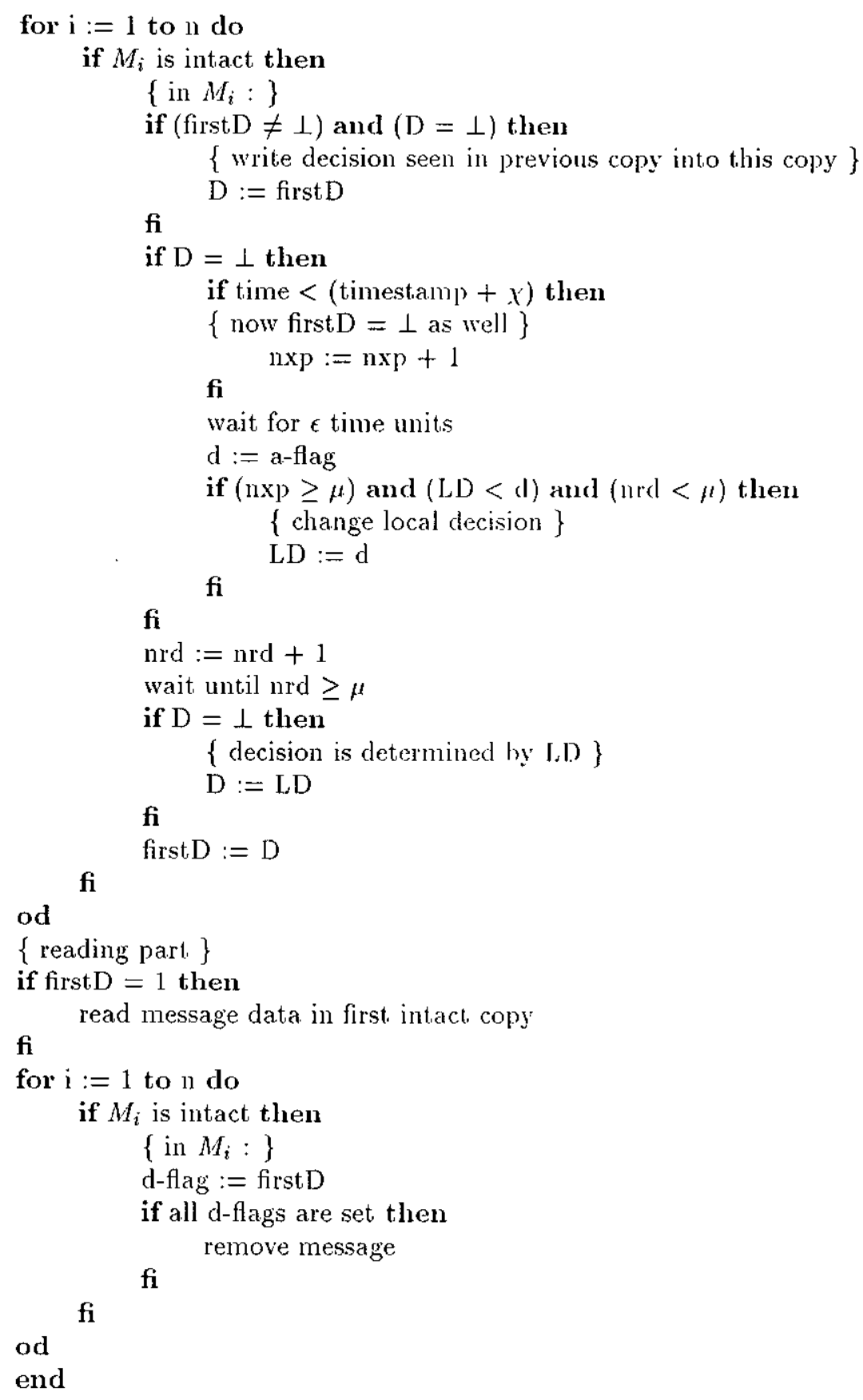

It is crucial that testing the values in the decision field and subsequentially updating them (lines $12-15$ and $30-33$, is executed as an atomic step. An interleaving of these operations by two receivers may cause LD to change after nrd has reached a value of $\mu$.

It should be noted, furthermore, that, in the pseudo-code above there are no provisions for the crash of a copy while the receiver is operating on it. If this happens, the receiver should move on to the next copy.

The two decision fields D and LD may seem superfluous, but they are necessary for consistency. Suppose a faulty sender writes a message in all copies, and pauses before having 
set any a-flag. When the intact receivers are executing the Receive on $M_{2}$, the sender resumes action. In $M_{1}$, the decision will be 0 , as the reccivers see a cleared a-flag. Assume, furthermore, that there is a receiver that has not seen the decision in $M_{1}$ (because of a crash of that copy). In $M_{2}$, this receiver may set LD to 1 , if it finds the a-flag set. Without the $\mathrm{D}$ field to override $\mathrm{LD}$, this scenario leads to different group decisions in $M_{1}$ and $M_{2}$, which makes the protocol incorrect.

\subsubsection{Correctness of the second protocol}

Theorem 2 The second protocol satisfies the four requirements of consensus, validity, ordering and bounded time.

\section{Proof:}

Consensus: First, it should be observed that in each copy, the value of $\mathrm{D}$ is set only once. This value $\neq \perp$, since it is either being copicd from $L D$, or from a previous copy. The former case results in 0 or 1 , since $L D$ only takes on these values. The latter case follows from the former by induction on the copies.

Second, the variable first. D, which eventually holds a receiver's decision, only changes when the value of $D$ is read into it. This value is always read after $D$ has been written.

Let $M_{x}$ be the first intact copy from which all intact receivers read the value of $\mathrm{D}$ (i.e. execute "firstD := D" at line 34 ), and let $v$ be this value. By the failure assumption, there is such a copy. Since the value in firstD is overwritten each time the receiver executes "firstD :=D", it is not important what took place in the copies before $M_{x}$. In later copies, the value of $D$ is not stored in firstD until a quorum has been formed. Every receiver that does this must have read $v$ from $M_{x}$, and will therefore write $v$ into D. Hence, firstD does not change after $M_{x}$; all intact receivers decide on $v$.

Validity: If the sender is intact, any intact receiver will increase nxp in the first intact copy. Due to the subsequent delay of $\epsilon, n x p$ will be $\geq \mu$ when the first intact receiver reads the a-flag. Since more than half of the receivers remain intact, a.t least one of them is among the receivers that form the guorum. This intact receiver finds the a-flag set and ensures that LD is set to 1 before incrementing nrd, so that the message is accepted.

Ordering: Receivers can safely process a message after local time $\tau+\chi+\epsilon$. If, afterwards, a message with an earlier timestamp is found in the quene, no intact receiver will increase nxp. Consequentially, nxp will not reach the value of $\mu ; L D$ remains equal to 0 , and the earlier message will not be accepted. Therefore, the timestanp ordering is consistent even in the presence of senders with timing failures.

Bounded time: In almost all steps of the Receive procedure, a receiver does not have to wait for other processes, Hence, these steps are exccuted in bounded time. The only point where a receiver is delayed for an unspecified amount of time is where it waits for a quorum to be formed. At this point, the presence of a majority of intact receivers is sufficient to draw a final decision within bounded time: even if all failed receivers are late, the intact receivers together form a quorum. After the slowest of the intact receivers has reached this stcp, a quorum must have becn formed, and all receivers can proceed. 


\section{Protocol analysis}

\subsection{Performance}

The table below gives some characteristics related to the performance of the protocols.

\begin{tabular}{|l|c|c|}
\hline & first protocol & second protocol \\
\hline \hline max. number of sender failures & any & any \\
max. number of receiver failures $(t)$ & any & $\left\lfloor\frac{N-1}{2}\right\rfloor$ \\
max. execution time for Send $\left(\Delta_{s}\right)$ & $n \delta_{s}+n \delta_{f}$ & $n \delta_{s}+n \delta_{f}$ \\
max. execution time for Receive $\left(\Delta_{r}\right)$ & $n\left(\delta_{d l}+\delta_{r}+\delta_{f d}+\epsilon\right)$ & $n\left(\delta_{d}+\delta_{r}+\delta_{f d}\right)+p+\epsilon$ \\
delivery latency & $\Delta_{s}+\epsilon$ & $\chi+\epsilon$ \\
\hline
\end{tabular}

Note that the maximum execution times for the two protocols are not directly comparable, since their values of $\delta_{s}$ etc. may differ.

As can be seen from the table, the maximum execution times are linearly dependent on the number of copies. Other factors such as the number of connected processes do not affect these figures. In practice however, the influence of these factors will probably be expressed in the maximum time for simple reading and writing of common memory, which we have taken as a constant.

\subsection{Comparison with other protocols}

Because the protocols use common memory for message transfer instead of the usual communication channels, it is hard to make a quantitative comparison with other protocols. For example, the efficiency of link-based protocols is usually expressed in the number of overhead messages. However, it is possible to compare some characteristics:

Tolerated failure classes. Most known protocols are only resilient to crash failures of processors, and to omission fajlures of the network. An exception is [Cris89], where three protocols are presented, providing resilience to omission, timing: and anthentication-detectable response failures, respectively.

Bounded execution time. Of the cited publications, only [Cris\$9] guarantees an upper bound on the execution time. The other references make no assumption about a maximum delivery delay on basic message transmission, hence the execution time for the entire protocol is unbounded. However, with the assumption of bounded delay, [Birm87] also satisfies the requirement. In [Chan84], a bound on worst-case exccution time is sacrificed for efficiency in average cases.

In [Cris89], the upper bound depends on the diameter of the communication network; in [Birm87], it would vary with the number of receivers. For our protocols, the most important variable in the maximum execution time is the number of mailbox copies. 
Delivery latency. The delivery latency is the time between the actual reception of a message at a destination processor, and the time at which it is made available to the receiving process. In our protocols, receivers are not, allowed to read a message immediately after it is written in a mailbox copy. Instead, they must wait until some time after the send operation has been completed.

In [Cris89], receivers must postpone processing a message until it is certain that every intact destination has received it. Cristian's first protocol, which can be compared to our first protocol, has a similar delivery latency. The latency of Cristian's second protocol, tolerating timing failures, varies with the product t. $\epsilon$. In the round-based protocol in [Birm87], destinations must delay the delivery of a message for up to three times the maximum communication delay. Furthermore, the protocol depends on the cooperation of all destinations to keep the delivery latency low. Finally, the protocol in [Chan84] has a delivery latency of zero, except when messages are received out of order.

Summarizing, we conclude that our protocols have properties similar to that of known (LANbased) protocols, while retajning the advantages of mailbox communication.

\section{Conclusion}

In this paper, two protocols for reliable multicast, with replicated mailboxes have been presented. The first protocol suffices if processes are fail-silent. The second protocol can also tolerate process timing failures.

The probability of failure of all copies of a nailbox was assumed to be zero; in practice, it can be reduced to a negligible value by creating a sufficient number of copies. Both protocols can tolerate an arbitrary number of sender failures. The number of receiver failures that can be tolerated differs: for the first protocol, it can be arbitrary, the second protocol requires that more than half of the receivers remains intact. Execution times are bounded, and vary only linearly with the number of copies.

As yet, the number of copies is determined at mailbox creation time, and does not change thereafter. The possibility to replace failed copies is a subject of further research. Future work also includes an implementation of the protocols on the EMPS system [Dijk92].

\section{A Protocol extensions}

\section{A.1 Dynamic multicast groups}

The protocols that were presented in the previous sections assumed that multicast groups are static. The set of receivers is thought to be known at mailbox creation time, and is only changed by a receiver crash. However, it is possible to make group membership dynamic. Two more primitives are needed: the Join procedure to add a receiver to the group, and the Leave procedure to remove a receiver.

We require that Joins and Leaves are consistent. That is, for a Join there must be a point in real time, after which every message that is sent contains the new receiver in its list of d-flags. Analogously, for each Leave there must be a point after which no message has a d-flag for that receiver.

To enable dynamic group membership, the receiver list must be modified. Each entry in that list not only contains the PID, but also two timestamps: an entry timestamp, indicating 
the time at which the receiver became a member, and an exit timestamp, containing the time at which the receiver left the group. Senders create a d-flag for a receiver if the timestamp of the message lies between the entry and exit timestamps (assuming an exit timestamp of infinity if the receiver has not yet left the group).

A Join operation consists of calculating the entry timestamp, and then adding an entry to the receiver list in every copy. The entry timestamp is determined as follows. If a process starts a Join operation at local time $\tau$, then at time $\tau+\Delta_{j}$ it has finished. Thus, for any message with a timestamp larger than $\tau+\Delta_{j}+\epsilon$ it is certain that the receiver is ready to read the message.

The exit timestamp is determined in an analogous way.

Join procedure A process that wants to join the multicast group must first ask the mailbox server for the location of the mailbox. If it is permitted to join the group, it gets references to the mailbox copies. Then it calculates its entry timestamp. After that, it adds its PID to the receiver list in each mailbox copy.

1 procedure Join

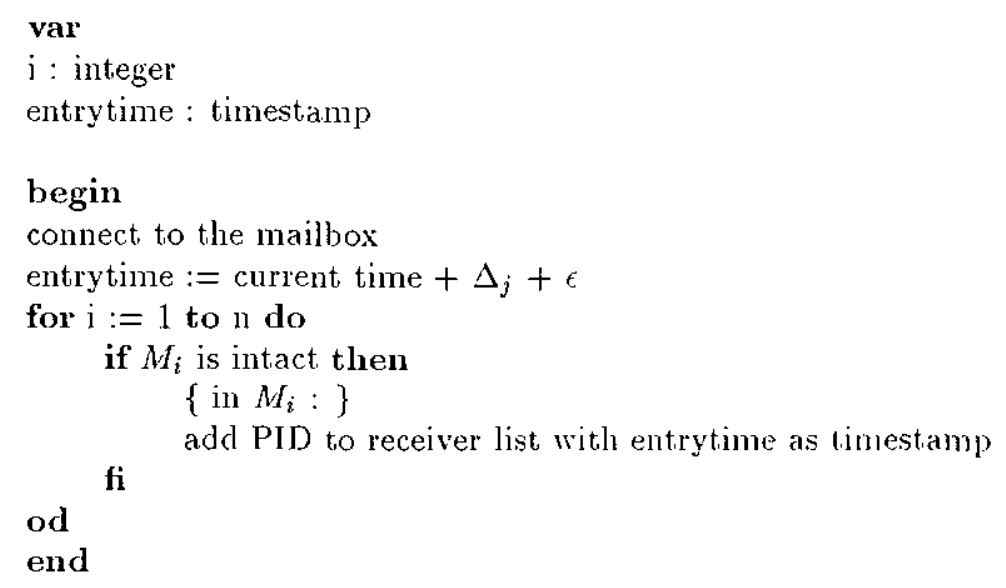

Leave procedure Leaving the multicast group is analogous to joining. When the process has determined the exit timestamp, it modifies its cntry in the receiver list. After completing the leave operation, the recciver must read the unread messages that have an earlier timestamp.

1 procedure Leave

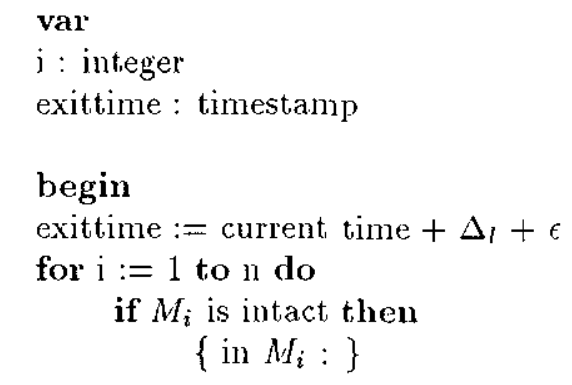




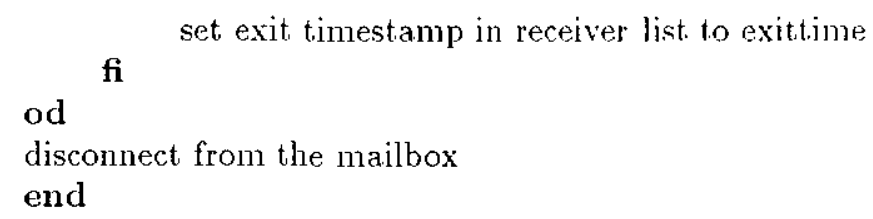

\section{A.1.1 Correctness of Join and Leave for the first protocol}

If senders and receivers are fail-silent, the correctness of Join and Leave operations follows from two claims, which are easy to verify:

Claim 1 If a receiver has completed its. Join. but not yet started a Leave operation, then every message with a timestamp $\geq$ the entry time contains a d-flag for that receiver.

Claim 2 If a receiver has completed its Leave operation, then every message with a timestamp $\geq$ the exit time does not contain a d-flag for that receiver.

\section{A.1.2 Suitability of Join and Leave for the second protocol}

If process timing failures are included in the failure hypothesis, the Join and Leave procedures are not suitable for the second protocol. The above claims hold no longer. That is, they are only valid for messages sent by intact senders. Problems may occur if the sender experiences timing failures. It may happen that in some message there is no d-flag for a certain receiver, even though the receiver has completed a Join (and is intact), and the message has a timestamp that is later than the entry time. This means that the receiver does not take part in the group decision. It may even be unable to read the message, since the message is deleted if all d-flags are set.

Fortunately, two minor modifications suffice to make the Join and Leave correct. First, a new receiver should "listen in". That is, it shonld read a message if the timestamp is later than its entry time, even if there is no d-flag. Absence of a d-flag only means that the receiver should not modify the decision field. Second, it must be ensured that such messages remain available to the receiver. This is done by postponing the removal of a message until $\Delta_{s}+\Delta_{r}+2 \epsilon$ after the timestamp (i.e. $\Delta_{r}+\epsilon$ time units after the receiving could be started). This time interval is enough for any intact receiver to finish the reading of the message.

\section{A.2 Inter-node multicast}

Typically, processors in a shared-memory system access the common memory through a bus. This is a limitation compared to LAN-based protocols, since the physical distance between processors on a common bus must be kept low. For some application such as process control, this is a serious disadvantage.

EMPS, the hardware on which DEDOS is being built. is not a pure shared-memory system. The highest level consists of norles, interconnected by a LAN. Each node contains a number of processors and memory-modules, connected to a common bus [Dijk92]. The multicast protocols as they have been described in this paper can only be used on EMPS if all senders and receivers are located on the same node.

It is possible to extend the protocols to perform inter-node multicast, i.e. to include "remote" processes in a multicast group. Each remote process must start a "slave" process 
which resides on the same node as the mailbox copies. Requests for operations are transmitted via the LAN to the slave process, which performs the actual multicast. After completion, the results are sent back to the remote process. If the LAN offers a reliable datagram service between the master and the slave, the multicast is also reliable. The bounded-time properties can be retained as well, provided that the datagram service has a bounded execution time.

\section{Acknowledgements}

This paper has benefited from the comments of Dieter Hammer, Henk Schepers, and especially Mathai Joseph, whose remarks have led to a considerable reduction of the complexity of the protocols.

\section{References}

[Birm87] K.P. Birman and T.A. Joseph: "Reliable communication in the presence of failures", ACM Transactions on Computer Systems, Vol. 5, No. 1, February 1987, pp. $47-76$.

[Chan84] J. Chang and N.F. Maxemchuk, "Reliable Broadcast. Protocols", ACM Transactions on Computer Systems, Vol. 2, No. 3,August 1984, pp. 251-273.

[Cris89] F. Cristian, H. Aghili, R. Strong and D. Dolev, "Atomic broadcast: from simple message diffusion to Byzanline agreement:". Technical Report R.J5244 (54244), IBM Research Lab, December 1989.

[Clae92] M.M.M.P.J. Claessen, "Membership service in DEDOS", Master's thesis, Eindhoven University of Technology, 1992.

[Cris91] F. Cristian, "Understanding fault-tolerant distributed systems", Communications of the ACM, Vol. 34, No. 2, February 1991, pp. 56-78.

[Dijk92] G.J.W van Dijk and M.J. van Gils, "Efficient, process migration in the EMPS multiprocessor", Proceedings of the $0^{\text {th }}$ International Parallel Processing Symposium, 1992 , pp. $58-66$.

[Garc89] H. Garcia-Molina and A. Spanster, "Message ordering in a multicast environment", Proceedings of the $9^{\text {th }}$ International Conference on Distributed Computing Systems (ICDCS), 1989, pp. 354-361.

[Stok92] P.D.V. v.d. Stok et al., "The dependable distributed operating system DEDOS", to be published as Eindhoven University of Technology Computing Science Note. 
In this series appeared:

91/01 D. Alstein

91/02 R.P. Nederpelt H.C.M. de Swart

91/03 J.P. Katoen L.A.M. Schoenmakers

91/04 E. v.d. Sluis A.F. v.d. Stappen

91/05 D. de Reus

91/06 K.M. van Hee

91/07 E.Poll

91/08 H. Schepers

91/09 W.M.P.v.d.Aalst

91/10 R.C.Backhouse

P.J. de Bruin

P. Hoogendijk

G. Malcolm

E. Voermans

J. v.d. Woude

91/11 R.C. Backhouse

P.J. de Bruin

G.Malcolm

E.Voermans

J. van der Woude

91/12 E. van der Sluis

91/13 F. Rietman

91/14 P. Lemmens

91/15 A.T.M. Aerts

K.M. van Hee

91/16 A.J.J.M. Marcelis

91/17 A.T.M. Aerts

P.M.E. de Bra

K.M. van Hee
Dynamic Reconfiguration in Distributed Hard Real-Time Systems, p. 14.

Implication. A survey of the different logical analyses "if...,then...", p. 26.

Parallel Programs for the Recognition of $P$-invariant Segments, p. 16.

Performance Analysis of VLSI Programs, p. 31.

An Implementation Model for GOOD, p. 18.

SPECIFICATIEMETHODEN, een overzicht, p. 20.

CPO-models for second order lambda calculus with recursive types and subtyping, p. 49.

Terminology and Paradigms for Fault Tolerance, p. 25.

Interval Timed Petri Nets and their analysis, p.53.

POLYNOMIAL RELATORS, p. 52.

Relational Catamorphism, p. 31.

A parallel local search algorithm for the travelling salesman problem, p. 12.

A note on Extensionality, p. 21.

The PDB Hypermedia Package. Why and how it was built, p. 63.

Eldorado: Architecture of a Functional Database Management System, p. 19.

An example of proving attribute grammars correct: the representation of arithmetical expressions by DAGs, p. 25 .

Transforming Functional Database Schemes to Relational Representations, p. 21. 
91/18 Rik van Geldrop

91/19 Erik Poll

91/20 A.E. Eiben

R.V. Schuwer

91/21 J. Coenen W.-P. de Roever J.Zwiers

91/22 G. Wolf

91/23 K.M. van Hee

L.J. Somers

M. Voorhoeve

91/24 A.T.M. Aerts

D. de Reus

91/25 P. Zhou

J. Hooman

R. Kuiper

91/26 P. de Bra

G.J. Houben

J. Paredaens

91/27 F. de Boer

C. Palamidessi

91/28 F. de Boer

91/29 H. Ten Eikelder

R. van Geldrop

91/30 J.C.M. Baeten

F.W. Vaandrager

91/31 H. ten Eikelder

91/32 P. Struik

91/33 w. v.d. Aalst

91/34 J. Coenen

91/35 F.S. de Boer

J.W. Klop

C. Palamidessi
Transformational Query Solving, p. 35.

Some categorical properties for a model for second order lambda calculus with subtyping, p. 21.

Knowledge Base Systems, a Formal Model, p. 21.

Assertional Data Reification Proofs: Survey and

Perspective, p. 18.

Schedule Management: an Object Oriented Approach, p. 26.

$\mathrm{Z}$ and high level Petri nets, p. 16.

Formal semantics for BRM with examples, p. 25.

A compositional proof system for real-time systems based on explicit clock temporal logic: soundness and complete ness, p. 52.

The GOOD based hypertext reference model, p. 12 .

Embedding as a tool for language comparison: On the CSP hierarchy, p. 17.

A compositional proof system for dynamic proces creation, p. 24.

Correctness of Acceptor Schemes for Regular Languages, p. 31.

An Algebra for Process Creation, p. 29.

Some algorithms to decide the equivalence of recursive types, p. 26.

Techniques for designing efficient parallel programs, $p$. 14.

The modelling and analysis of queueing systems with QNM-ExSpect, p. 23.

Specifying fault tolerant programs in deontic logic, p. 15.

Asynchronous communication in process algebra, p. 20. 


\begin{tabular}{|c|c|}
\hline $92 / 01$ & $\begin{array}{l}\text { J. Coenen } \\
\text { J. Zwiers } \\
\text { W.-P. de Roever }\end{array}$ \\
\hline $92 / 02$ & $\begin{array}{l}\text { J. Coenen } \\
\text { J. Hooman }\end{array}$ \\
\hline $92 / 03$ & $\begin{array}{l}\text { J.C.M. Baeten } \\
\text { J.A. Bergstra }\end{array}$ \\
\hline $92 / 04$ & J.P.H.W.v.d.Eijnde \\
\hline $92 / 05$ & J.P.H.W.v.d.Eijnde \\
\hline $92 / 06$ & $\begin{array}{l}\text { J.C.M. Baeten } \\
\text { J.A. Bergstra }\end{array}$ \\
\hline $92 / 07$ & R.P. Nederpelt \\
\hline $92 / 08$ & $\begin{array}{l}\text { R.P. Nederpelt } \\
\text { F. Kamareddine }\end{array}$ \\
\hline $92 / 09$ & R.C. Backhouse \\
\hline $92 / 10$ & P.M.P. Rambags \\
\hline $92 / 11$ & $\begin{array}{l}\text { R.C. Backhouse } \\
\text { J.S.C.P.v.d.Woude }\end{array}$ \\
\hline $92 / 12$ & F. Kamareddine \\
\hline $92 / 13$ & F. Kamareddine \\
\hline $92 / 14$ & J.C.M. Baeten \\
\hline $92 / 15$ & F. Kamareddine \\
\hline $92 / 16$ & R.R. Seljée \\
\hline
\end{tabular}

92/17 W.M.P. van der Aalst

92/18 R.Nederpelt F. Kamareddine

92/19 J.C.M.Baeten J.A.Bergstra S.A.Smolka

92/20 F.Kamareddine

92/21 F.Kamareddine
A note on compositional refinement, p. 27.

A compositional semantics for fault tolerant real-time systems, p. 18.

Real space process algebra, p. 42.

Program derivation in acyclic graphs and related problems, p. 90.

Conservative fixpoint functions on a graph, p. 25.

Discrete time process algebra, p.45.

The fine-structure of lambda calculus, p. 110.

On stepwise explicit substitution, p. 30 .

Calculating the Warshall/Floyd path algorithm, p. 14.

Composition and decomposition in a CPN model, p. 55.

Demonic operators and monotype factors, p. 29.

Set theory and nominalisation, Part I, p.26.

Set theory and nominalisation, Part II, p.22.

The total order assumption, p. 10.

A system at the cross-roads of functional and logic programming, p.36.

Integrity checking in deductive databases; an exposition, p.32.

Interval timed coloured Petri nets and their analysis, $\mathrm{p}$. 20.

A unified approach to Type Theory through a refined lambda-calculus, p. 30.

Axiomatizing Probabilistic Processes:

ACP with Generative Probabilities, p. 36.

Are Types for Natural Language? P. 32.

Non well-foundedness and type freeness can unify the interpretation of functional application, p. 16. 


\begin{tabular}{|c|c|c|}
\hline $92 / 22$ & $\begin{array}{l}\text { R. Nederpelt } \\
\text { F.Kamareddine }\end{array}$ & A useful lambda notation, p. 17 . \\
\hline $92 / 23$ & $\begin{array}{l}\text { F.Kamareddine } \\
\text { E.Klein }\end{array}$ & Nominalization, Predication and Type Containment, p. 40. \\
\hline $92 / 24$ & $\begin{array}{l}\text { M.Codish } \\
\text { D.Dams } \\
\text { Eyal Yardeni }\end{array}$ & $\begin{array}{l}\text { Bottum-up Abstract Interpretation of Logic Programs, } \\
\text { p. } 33 .\end{array}$ \\
\hline $92 / 25$ & E.Poll & A Programming Logic for $F \omega$, p. 15. \\
\hline $92 / 26$ & $\begin{array}{l}\text { T.H.W.Beelen } \\
\text { W.J.J.Stut } \\
\text { P.A.C.Verkoulen }\end{array}$ & $\begin{array}{l}\text { A modelling method using MOVIE and SimCon/ExSpect, } \\
\text { p. } 15 .\end{array}$ \\
\hline $92 / 27$ & $\begin{array}{l}\text { B. Watson } \\
\text { G. Zwaan }\end{array}$ & $\begin{array}{l}\text { A taxonomy of keyword pattern matching algorithms, } \\
\text { p. } 50 \text {. }\end{array}$ \\
\hline $93 / 01$ & R. van Geldrop & $\begin{array}{l}\text { Deriving the Aho-Corasick algorithms: a case study into } \\
\text { the synergy of programming methods, p. } 36 \text {. }\end{array}$ \\
\hline $93 / 02$ & T. Verhoeff & A continuous version of the Prisoner's Dilemma, p. 17 \\
\hline $93 / 03$ & T. Verhoeff & Quicksort for linked lists, p. 8. \\
\hline $93 / 04$ & $\begin{array}{l}\text { E.H.L. Aarts } \\
\text { J.H.M. Korst } \\
\text { P.J. Zwietering }\end{array}$ & Deterministic and randomized local search, p. 78 . \\
\hline $93 / 05$ & $\begin{array}{l}\text { J.C.M. Baeten } \\
\text { C. Verhoef }\end{array}$ & $\begin{array}{l}\text { A congruence theorem for structured operational } \\
\text { semantics with predicates, p. } 18 \text {. }\end{array}$ \\
\hline $93 / 06$ & J.P. Veltkamp & On the unavoidability of metastable behaviour, p. 29 \\
\hline $93 / 07$ & P.D. Moerland & Exercises in Multiprogramming, p. 97 \\
\hline $93 / 08$ & J. Verhoosel & $\begin{array}{l}\text { A Formal Deterministic Scheduling Model for Hard Real- } \\
\text { Time Executions in DEDOS, p. } 32 \text {. }\end{array}$ \\
\hline $93 / 09$ & K.M. van Hee & $\begin{array}{l}\text { Systems Engineering: a Formal Approach } \\
\text { Part I: System Concepts, p. } 72 \text {. }\end{array}$ \\
\hline $93 / 10$ & K.M. van Hee & $\begin{array}{l}\text { Systems Engineering: a Formal Approach } \\
\text { Part II: Frameworks, p. } 44 .\end{array}$ \\
\hline $93 / 11$ & K.M. van Hee & $\begin{array}{l}\text { Systems Engineering: a Formal Approach } \\
\text { Part III: Modeling Methods, p. } 101 .\end{array}$ \\
\hline $93 / 12$ & K.M. van Hee & $\begin{array}{l}\text { Systems Engineering: a Formal Approach } \\
\text { Part IV: Analysis Methods, p. } 63 \text {. }\end{array}$ \\
\hline $93 / 13$ & K.M. van Hee & $\begin{array}{l}\text { Systems Engineering: a Formal Approach } \\
\text { Part V: Specification Language, p. } 89 .\end{array}$ \\
\hline
\end{tabular}




\begin{tabular}{|c|c|c|}
\hline $92 / 22$ & $\begin{array}{l}\text { R. Nederpelt } \\
\text { F.Kamareddine }\end{array}$ & A useful lambda notation, p. 17 \\
\hline $92 / 23$ & $\begin{array}{l}\text { F.Kamareddine } \\
\text { E.Klein }\end{array}$ & Nominalization, Predication and Type Containment, p. 40. \\
\hline $92 / 24$ & $\begin{array}{l}\text { M.Codish } \\
\text { D.Dams } \\
\text { Eyal Yardeni }\end{array}$ & $\begin{array}{l}\text { Bottum-up Abstract Interpretation of Logic Programs, } \\
\text { p. } 33 .\end{array}$ \\
\hline $92 / 25$ & E.Poll & A Programming Logic for $F \omega$, p. 15. \\
\hline $92 / 26$ & $\begin{array}{l}\text { T.H.W.Beelen } \\
\text { W.J.J.Stut } \\
\text { P.A.C.Verkoulen }\end{array}$ & $\begin{array}{l}\text { A modelling method using MOVIE and SimCon/ExSpect, } \\
\text { p. } 15 \text {. }\end{array}$ \\
\hline $92 / 27$ & $\begin{array}{l}\text { B. Watson } \\
\text { G. Zwaan }\end{array}$ & $\begin{array}{l}\text { A taxonomy of keyword pattern matching algorithms, } \\
\text { p. } 50 .\end{array}$ \\
\hline $93 / 01$ & R. van Geldrop & $\begin{array}{l}\text { Deriving the Aho-Corasick algorithms: a case study into } \\
\text { the synergy of programming methods, p. } 36 \text {. }\end{array}$ \\
\hline $93 / 02$ & T. Verhoeff & A continuous version of the Prisoner's Dilemma, p. 17 \\
\hline $93 / 03$ & T. Verhoeff & Quicksort for linked lists, p. 8. \\
\hline $93 / 04$ & $\begin{array}{l}\text { E.H.L. Aarts } \\
\text { J.H.M. Korst } \\
\text { P.J. Zwietering }\end{array}$ & Deterministic and randomized local search, p. 78 . \\
\hline $93 / 05$ & $\begin{array}{l}\text { J.C.M. Baeten } \\
\text { C. Verhoef }\end{array}$ & $\begin{array}{l}\text { A congruence theorem for structured operational } \\
\text { semantics with predicates, p. } 18 \text {. }\end{array}$ \\
\hline $93 / 06$ & J.P. Veltkamp & On the unavoidability of metastable behaviour, p. 29 \\
\hline $93 / 07$ & P.D. Moerland & Exercises in Multiprogramming, p. 97 \\
\hline $93 / 08$ & J. Verhoosel & $\begin{array}{l}\text { A Formal Deterministic Scheduling Model for Hard Real- } \\
\text { Time Executions in DEDOS, p. } 32 .\end{array}$ \\
\hline $93 / 09$ & K.M. van Hee & $\begin{array}{l}\text { Systems Engineering: a Formal Approach } \\
\text { Part I: System Concepts, p. } 72 .\end{array}$ \\
\hline $93 / 10$ & K.M. van Hee & $\begin{array}{l}\text { Systems Engineering: a Formal Approach } \\
\text { Part II: Frameworks, p. } 44 .\end{array}$ \\
\hline $93 / 11$ & K.M. van Hee & $\begin{array}{l}\text { Systems Engineering: a Formal Approach } \\
\text { Part III: Modeling Methods, p. } 101 .\end{array}$ \\
\hline $93 / 12$ & K.M. van Hee & $\begin{array}{l}\text { Systems Engineering: a Formal Approach } \\
\text { Part IV: Analysis Methods, p. } 63 \text {. }\end{array}$ \\
\hline $93 / 13$ & K.M. van Hee & $\begin{array}{l}\text { Systems Engineering: a Formal Approach } \\
\text { Part V: Specification Language, p. } 89 .\end{array}$ \\
\hline $93 / 14$ & $\begin{array}{l}\text { J.C.M. Baeten } \\
\text { J.A. Bergstra }\end{array}$ & $\begin{array}{l}\text { On Sequential Composition, Action Prefixes and } \\
\text { Process Prefix, p. } 21 .\end{array}$ \\
\hline
\end{tabular}


93/15 J.C.M. Baeten

J.A. Bergstra

R.N. Bol

93/16 H. Schepers

J. Hooman
A Real-Time Process Logic, p. 31.

A Trace-Based Compositional Proof Theory for Fault Tolerant Distributed Systems, p. 27 